\title{
Numerical Solutions of Coupled Systems of Fractional Order Partial Differential Equations
}

\author{
Yongjin $\mathrm{Li}^{1}$ and Kamal Shah ${ }^{2}$ \\ ${ }^{1}$ Department of Mathematics, Sun Yat-Sen University, Guangzhou, China \\ ${ }^{2}$ Department of Mathematics, University of Malakand, Chakdara Dir (L), Khyber Pakhtunkhwa, Pakistan \\ Correspondence should be addressed to Kamal Shah; kamalshah408@gmail.com
}

Received 26 May 2017; Revised 11 July 2017; Accepted 16 July 2017; Published 20 September 2017

Academic Editor: Rehana Naz

Copyright (c) 2017 Yongjin Li and Kamal Shah. This is an open access article distributed under the Creative Commons Attribution License, which permits unrestricted use, distribution, and reproduction in any medium, provided the original work is properly cited.

We develop a numerical method by using operational matrices of fractional order integrations and differentiations to obtain approximate solutions to a class of coupled systems of fractional order partial differential equations (FPDEs). We use shifted Legendre polynomials in two variables. With the help of the aforesaid matrices, we convert the system under consideration to a system of easily solvable algebraic equation of Sylvester type. During this process, we need no discretization of the data. We also provide error analysis and some test problems to demonstrate the established technique.

\section{Introduction}

Recently fractional calculus has attracted the attention of many researchers as it has many applications in various disciplines of applied sciences and engineering (we refer to [1-4] and the references therein). In fact most of the engineering and physical processes can be modeled and well explained by using a system of fractional order differential and integral equations more realistically as compared to system of conventional differential and integral equations, (see $[1,5-$ 15] and the references therein). The area of fractional calculus devoted to the existence and uniqueness of positive solution to (FDEs) and (FPDEs) is well studied and a lot of research work is available on it (we refer to [16-20]). In the last two decades, the area dealing with numerical solutions of FDEs and FPDEs has attracted the attention of many researchers. Researchers are taking keen interests in developing numerical procedures for FPDEs. Here, it is remarkable that most of the problems of applied sciences can be modeled by using PDEs and their systems. The study of coupled systems of PDEs can widely be found in engineering and biomechanics and other disciplines. For example, in biomechanics when modeling the phenomena of electrical activity in the heart, the resulting equations are coupled systems of PDEs (see, e.g.,
[21-23]). Coupled systems of PDEs also occur in modeling of some chemical and material engineering processes such as system containing a continuous stirred tank reactor (CSTR) and a plug flow reactor (PFR) in series (see [24, 25]). Various applications of coupled systems can be found in solid state physics and mechanics; for example, dynamics of multideformable bodies coupled by standard light fractional order discrete continuum layers is described by coupled systems of fractional order partial differential equations [7, 26-28]. The coupled systems of PDEs also appear in modeling of some important electromagnetic and gravitational problems, (see, e.g., $[29,30])$.

Most of the FPDEs do not have exact analytical solutions; therefore researchers need some appropriate numerical technique for the approximate solutions of such types (FPDEs). For the numerical solutions numerous techniques are available in the literature; for example, some of them are eigenvector expansion, Adomian decomposition method (ADM), fractional differential transform method (FDTM) $[31,32]$, generalized block pulse operational matrix method [33], and so on. In recent years many attentions have been devoted to develop operational matrices for fractional order differentiation and integrations. Such types of operational matrices are obtained by using Haar wavelets, Legendre 
wavelets, Sine Cosine, Chebyshev wavelets, and so on. Based on these operational matrices, some approximate methods for numerical solutions to FDEs have been developed (we refer to [34-36] and the references therein). In [37], the authors established a new numerical scheme based on the Haar wavelet for solving (FPDEs). More recently, the authors [38] developed some new results related to the Jacobi polynomials for operational matrices. All the operational matrices methods are used to solve FDEs and FPDEs. As much as we know they are not well studied and neither is properly applied to solve coupled systems of PFDEs. To the best of our knowledge very few articles are devoted to the numerical solutions of coupled systems of integral equations and ordinary and partial differential equations of fractional order; for details see [39-41].

This manuscript is devoted to bring out the numerical solutions to a coupled system of FPDEs on an unbounded domain. The concerned coupled system contains mixed partial order derivative with respect to $x, y$ as given by

$$
\begin{aligned}
& { }^{c} D_{x}^{p} U(x, y)+\omega_{1}{ }^{c} D_{x y}^{p_{1}} V(x, y)+\omega_{2}{ }^{c} D_{y}^{q_{1}} U(x, y) \\
& \quad=\theta(x, y), \\
& { }^{c} D_{x}^{p^{p}} V(x, y)+\rho_{1}{ }^{c} D_{x y}^{p_{2}} U(x, y)+\rho_{2}{ }^{c} D_{y}^{q_{2}} V(x, y) \\
& \quad=\psi(x, y),
\end{aligned}
$$

corresponding to the conditions

$$
\begin{aligned}
U^{(i)}(0, y) & =\theta_{i}(y), \\
V^{(i)}(0, y) & =\psi_{i}(y), \\
& i=1 \cdots m,
\end{aligned}
$$

where ${ }^{c} D_{z}^{p}=\partial^{p} / \partial z^{p}$ represents partial derivative, $p \in(k-$ $1, k], k-2<p_{i}, q_{i} \leq k-1$, the real constants are denoted by $\omega_{i}, \rho_{i}(i=1,2), \theta_{i}, \psi_{i}$, and

$$
\begin{aligned}
U(x, y), V(x, y), \theta(x, y), \psi(x, y) & \\
& \in C([0,1] \times[0,1]) .
\end{aligned}
$$

It is remarkable that the proposed system contains the coupled system investigated in [38] as a special case. If we take $\theta(x, y)=\psi(x, y)=0$ together with $\omega_{1}=0, \omega_{2}=$ 1 and $\rho_{1}=0, \rho_{2}=1$, the proposed system becomes a coupled system of Laplace equations of fractional order by taking $p=q_{1}=q_{2} \in(1,2]$. Further, if we involve the external (source) terms and consider $\omega_{1}=\rho_{1}=0$, the proposed coupled system assumes the form of coupled system of Poisson's equation of arbitrary order. Poisson's equations have many applications in physics as well as in other various disciplines including electrostatics, mechanical engineering, and theoretical physics. For example, it has been applied to describe the potential energy field caused by a given charge or mass density distribution (see for details [42]).

With the use of shifted Legendre polynomials in two variables, some operational matrices corresponding to fractional order differentiations and integrations are developed. Thanks to these operational matrices, the coupled system under consideration is transformed to a system of Sylvester type algebraic equations. Here, we remark that no discretization like for Tau-collocations method is required. By doing so, the proposed system (1) corresponding to the initial conditions is transformed to a system of Sylvester type algebraic equations. Our proposed method is simple and there is no computational complexity in the resulting algebraic system to solve. Further, we remark that our present method is a numerical method based on shifted Jacobi polynomials while the method discussed in [43] is an iterative method based on monotone iterative technique. Further we can easily establish a simple relationship for convergence of the propped method. The aforesaid procedure has now recently being applied for some nonlinear fractional order differential equations and some fruitful results were obtained; for details see [44]. In our future work we will use operational matrices method to compute approximate solutions of nonlinear FPDEs and their system.

The manuscript is organized as follows: Section 2 is concerning some basic definitions and preliminaries results related to fractional calculus and Legendre polynomials which are needed to form the proposed method. Section 3 is concerning the operational matrices corresponding to the fractional order derivatives and integration. Further, Section 4 is related to the application of the operational matrices to covert coupled system (1) to the corresponding system of algebraic equations. Section 5 is devoted to the numerical examples, and in the last section, we have provided a brief conclusion.

\section{Preliminaries}

Definition 1. The Mittag-Leffler function subjected to one parameter is recalled by

$$
E_{p}(x)=\sum_{n=0}^{\infty} \frac{x^{n}}{\Gamma(n p+1)} .
$$

The extension of (4) to two parameters is given as

$$
E_{p, q}(x)=\sum_{n=0}^{\infty} \frac{x^{n}}{\Gamma(n p+q)} .
$$

Definition 2 (see $[45,46])$. The fractional integral of Riemann-Liouville type of order $p \in(0, \infty)$ of a function $\theta \in L^{1}([0, a], \mathfrak{R})$ is defined as

$$
I_{a+}^{p} \theta(x)=\frac{1}{\Gamma(p)} \int_{a}^{x}(x-\eta)^{p-1} \theta(\eta) d \eta,
$$

provided that the right-hand side is pointwise defined on $(0, \infty)$.

Definition 3. For a given function $\theta(x, y)$, the Caputo fractional derivative of order $p$ is defined as

$$
\begin{array}{r}
{ }^{c} D^{p} \theta(x, y)=\frac{1}{\Gamma(k-p)} \int_{a}^{x}(x-\eta)^{k-p-1} \theta^{(k)}(\eta, y) d \eta, \\
p \in(k-1, k], k \in N,
\end{array}
$$


provided that the right side is pointwise defined on $(a, \infty)$, where $k=[p]+1$.

Hence, it follows that

$$
\begin{aligned}
{ }^{c} D^{p} x^{n} & =\frac{\Gamma(1+n)}{\Gamma(1+n-p)} x^{n-p}, \\
I^{p} x^{n} & =\frac{\Gamma(1+n)}{\Gamma(1+n+p)} x^{n+p}, \\
& { }^{c} D^{p} C=0, \text { for a constant } C .
\end{aligned}
$$

Theorem 4 (see [45]). The solution of FDE

$$
{ }^{c} D_{x}^{p} U(x, y)=h(x), \quad k-1<p \leq k,
$$

is given by

$$
U(x, y)=I^{p} h(x)+e_{0}+e_{1} x+\cdots+e_{k-1} x^{k-p},
$$

where each $e_{i}$ is a real number for $i=0,1,2, \ldots, k-1$, where $k=[p]+1$, such that $[p]$ represents integer part of $p$.

Remark 5. We will use Caputo derivative throughout this paper, because Caputo fractional derivative has clear geometrical representations like classical derivative. Further, it turns out that the Riemann-Liouville derivatives have certain disadvantages when trying to model real-world phenomena with fractional differential equations. For further details, see $[45,47]$.

2.1. The Shifted Legendre Polynomials. The well-known Legendre polynomials described over $[-1,1]$ are representing as

$$
\begin{aligned}
\mathscr{P}_{i+1}(t) & =\frac{2 i+1}{i+1} t \mathscr{P}_{i}(t)-\frac{i}{i+1} \mathscr{P}_{i-1}(t), \\
& i=1,2,3, \ldots, \text { where } \mathscr{P}_{0}(t)=1, \mathscr{P}_{1}(t)=t .
\end{aligned}
$$

The transformation $x=(t+1) / 2$ transforms the interval $[-1,1]$ to $\mathbf{J}=[0,1]$ and the corresponding polynomials known as shifted Legendre polynomials are given as

$$
\mathfrak{\Omega}_{i}(x)=\sum_{n=0}^{i} \frac{(-1)^{i+n} \Gamma(i+n+1)}{\Gamma(i-n+1) \Gamma^{2}(n+1)} x^{n},
$$

The orthogonality condition is

$$
i=1,2,3, \ldots \text {. }
$$

$$
\int_{0}^{1} \mathfrak{Q}_{i}(x) \mathfrak{Q}_{j}(x) d x= \begin{cases}\frac{1}{2 i+1}, & \text { if } i \neq j, \\ 0, & \text { if } i=j .\end{cases}
$$

Therefore, any function $\theta(x) \in C(\mathbf{J})$ can be approximated in terms of shifted Legendre polynomials as

$$
\begin{aligned}
& \theta(x) \approx \sum_{\mu=0}^{m} \mathscr{C}_{\mu} \mathfrak{Q}_{\mu}(x), \\
& \text { where } \mathscr{C}_{\mu}=\left\langle\theta(x), \mathfrak{Q}_{\mu}(x)\right\rangle=(2 \mu+1) \int_{0}^{1} \theta(x) \mathfrak{Q}_{\mu}(x) d x .
\end{aligned}
$$

In matrix form, we may write (14) as

$$
\theta(x)=\mathbf{A}_{K}^{T} \Upsilon_{K^{2}}(x, y), \quad \text { where } K=m+1,
$$

where $\mathbf{A}_{K}$ is the matrix of functions while $\Upsilon_{K^{2}}(x, y)$ is $K$ term column matrix. The shifted Legendre polynomials of order $K$ for two dimensions are recalled by

$$
\begin{aligned}
\mathfrak{R}_{k}(x, y)=\mathfrak{Q}_{\mu}(x) & \mathfrak{Q}_{\nu}(y), \\
k & =K \mu+\nu+1, \mu, \nu=0,1,2, \ldots, m
\end{aligned}
$$

and the corresponding orthognathic relation is provided as

$$
\begin{aligned}
& \int_{0}^{1} \int_{0}^{1} \mathfrak{Q}_{\mu_{1}}(x) \mathfrak{Q}_{v_{1}}(y) \mathfrak{Q}_{\mu_{2}}(x) \mathfrak{Q}_{v_{2}}(y) d x d y \\
& \quad= \begin{cases}\frac{1}{(2 \mu+1)(2 v+1)}, & \text { if } \mu_{1}=\mu_{2}, \quad v_{1}=v_{2}, \\
0, & \text { if } \mu_{1} \neq \mu_{2}, \quad v_{1} \neq v_{2} .\end{cases}
\end{aligned}
$$

Therefore any function $U(x, y) \in C(\mathbf{J} \times \mathbf{J})$ can be approximated in terms of $\mathfrak{\Omega}_{k}(x, y)$ as

$$
U(x, y) \approx \sum_{\mu=0}^{m} \sum_{\nu=0}^{m} \mathfrak{s}_{\mu \nu} \mathbf{Q}_{\mu}(x) \mathbf{Q}_{\nu}(y),
$$

where

$$
\begin{aligned}
& \mathfrak{F}_{\mu \nu}=(2 \mu+1)(2 \nu+1) \\
& \text { - } \int_{0}^{1} \int_{0}^{1} U(x, y) \mathfrak{\Omega}_{\mu}(x) \mathfrak{\Omega}_{v}(y) d x d y .
\end{aligned}
$$

In matrix notation, we may write (18) as

$$
U(x, y) \approx \sum_{k=1}^{K^{2}} \mathfrak{c}_{\mu \nu} \mathbf{\Omega}_{n}(x, y)=\mathbf{A}_{K^{2}}^{T} \Upsilon_{K^{2}}(x, y)
$$

where the column vector of coefficients with order $K^{2} \times 1$ is represented as $\mathbf{A}_{K^{2}}, k=K \mu+\nu+1$. Also column vector of order $K^{2} \times 1$ is represented by $\Upsilon_{K^{2}}(x, y)$ and provided as

$$
\Upsilon_{K^{2}}(x, y)=\left[\begin{array}{llllllll}
\varphi_{11}(x, y) & \cdots & \varphi_{1 K}(x, y) & \varphi_{21}(x, y) & \cdots & \varphi_{2 K}(x, y) & \cdots & \varphi_{K K}(x, y)
\end{array}\right]^{T},
$$

where $\varphi_{i+1, j+1}(x, y)=\left(\mathfrak{Q}_{i}(x)\right)\left(\mathfrak{Q}_{j}(y)\right), i, j=0,1,2, \ldots, m$. 
2.2. Maximum Absolute Error ([48]). Consider a sufficiently smooth function $U(x, y)$ on $\mathbf{J} \times \mathbf{J}$; then the error in the numerical solutions is provided as

$$
\begin{aligned}
& \left\|U(x, y)-\mathfrak{\Omega}_{(K, K)}(x, y)\right\|_{2} \\
& \quad \leq\left(\mathfrak{c}_{1}+\mathfrak{c}_{2}+\mathfrak{c}_{3} \frac{1}{K^{K+1}}\right) \frac{1}{K^{K+1}},
\end{aligned}
$$

where

$$
\begin{aligned}
& \mathfrak{F}_{1}=\frac{1}{4} \max _{(x, y) \in \mathbf{J} \times \mathbf{J}}\left|\frac{\partial^{K+1}}{\partial x^{K+1}} U(x, y)\right|, \\
& \mathfrak{F}_{2}=\frac{1}{4} \max _{(x, y) \in \mathbf{J} \times \mathbf{J}}\left|\frac{\partial^{K+1}}{\partial y^{K+1}} U(x, y)\right|, \\
& \mathfrak{F}_{3}=\frac{1}{16} \max _{(x, y) \in \mathbf{J} \times \mathbf{J}}\left|\frac{\partial^{2 K+2}}{\partial x^{K+1} \partial y^{K+1}} U(x, y)\right| .
\end{aligned}
$$

The above relation also holds at points $\left(x_{i}, y_{i}\right)$ for interpolating polynomial $\mathfrak{\Omega}_{(K, K)}(x, y)$.

\section{Operational Matrices of Integrations and Differentiations}

Extending the notion of the operational matrices of fractional order integration and differentiation from one dimension
$[36,49]$ to two dimensions, the construction of the aforesaid operational matrices is provided as follows.

Theorem 6. The operational matrix corresponding to fractional integration of order $p$ of $\Upsilon_{K^{2}}(x, y)$ defined in (21) with respect to $x$ is provided as

$$
I_{x}^{p}\left[\Upsilon_{K^{2}}(x, y)\right] \simeq \mathbf{H}_{K^{2} \times K^{2}}^{(p, x)} \Upsilon_{K^{2}}(x, y),
$$

where $\mathbf{H}_{K^{2} \times K^{2}}^{(p, x)}$ is the operational matrix of fractional integration given as

$$
\begin{aligned}
& \mathbf{H}_{K^{2} \times K^{2}}^{(p, x)} \\
& =\left(\begin{array}{cccccc}
¥_{1,1, n} & ¥_{1,2, n} & \cdots & ¥_{1, u, n} & \cdots & ¥_{1, K^{2}, n} \\
¥_{2,1, n} & ¥_{2,2, n} & \cdots & \Omega_{2, u, n} & \cdots & ¥_{2, K^{2}, n} \\
\vdots & \vdots & \vdots & \vdots & \vdots & \vdots \\
¥_{v, 1, n} & ¥_{v, 2, n} & \cdots & ¥_{v, u, n} & \cdots & ¥_{v, K^{2}, n} \\
\vdots & \vdots & \vdots & \vdots & \vdots & \vdots \\
¥_{K^{2}, 1, n} & ¥_{K^{2}, 2, n} & \cdots & ¥_{K^{2}, u, n} & \cdots & ¥_{K^{2}, K^{2}, n}
\end{array}\right),
\end{aligned}
$$

$u=K i+j+1, \quad v=K \mu+v+1, ¥_{v, u, n}=\prod_{i, j, \mu, v, n}$ for $i, j, v, \mu=$ $0,1,2, \ldots, m$,

$$
\mathscr{E}_{i, j, \mu, \nu, n}=\sum_{n=0}^{\mu} \delta_{i, \nu}(2 i+1) \sum_{l=0}^{i} \frac{(-1)^{i+l+\mu+n} \Gamma(i+l+1) \Gamma(\mu+n+1)}{\Gamma(i-l+1) \Gamma^{2}(l+1) \Gamma(\mu-n+1) \Gamma(n+p+1)(n+l+p+1)}, \quad \delta_{i, \nu}= \begin{cases}0, & \text { if } i \neq v \\ 1, & \text { if } i=v\end{cases}
$$

Proof. Consider $\mathfrak{Q}_{k}(x, y)$ as given in (16); then the arbitrary order integral with order $p$ of $\mathfrak{\Omega}_{k}(x, y)$ corresponding to $x$ is provided as

$$
\begin{aligned}
I_{x}^{p} \mathfrak{Q}_{k}(x, y) & =I_{x}^{p} \mathfrak{Q}_{\mu}(x) \mathfrak{Q}_{v}(y) \\
& =\sum_{n=0}^{\mu} \frac{(-1)^{\mu+n} \Gamma(\mu+n+1)}{\Gamma(\mu-n+1) \Gamma^{2}(n+1)} I_{x}^{p} x^{n} \mathfrak{Q}_{v}(y),
\end{aligned}
$$

which gives that

$$
\begin{aligned}
& I_{x}^{p} \mathfrak{Q}_{\mu}(x) \mathfrak{Q}_{\nu}(y) \\
& =\sum_{n=0}^{\mu} \frac{(-1)^{\mu+n} \Gamma(\mu+n+1)}{\Gamma(\mu-n+1) \Gamma^{2}(n+1) \Gamma(n+p+1)} x^{n+p} P_{\nu}(y) .
\end{aligned}
$$

Further approximating $x^{n+p} \boldsymbol{Q}_{v}(y)$ in terms of shifted Legendre polynomials, we have

$$
x^{n+p} \mathfrak{Q}_{\nu}(y) \approx \sum_{i=0}^{m} \sum_{j=0}^{m} \mathscr{E}_{i, j} \mathfrak{Q}_{i}(x) \mathfrak{\Omega}_{j}(y)
$$

where $\mathscr{E}_{i, j}=(2 i+1)(2 j+1) \int_{0}^{1} \int_{0}^{1} x^{n+p} \mathfrak{Q}_{\nu}(y) \mathfrak{Q}_{i}(x) \mathfrak{Q}_{j}(y) d x d y$. Thanks to orthogonality condition, we have

$$
\begin{aligned}
& \mathscr{E}_{i, j} \\
& = \begin{cases}0, & \text { if } j \neq v, \\
(2 i+1) \sum_{l=0}^{j} \frac{(-1)^{i+l} \Gamma(i+l+1)}{\Gamma(i-l+1) \Gamma^{2}(l+1)(n+l+p+1)}, & \text { if } j=v\end{cases} \\
& =\delta_{i, \nu}(2 i+1) \sum_{l=0}^{i} \frac{(-1)^{i+l} \Gamma(i+l+1)}{\Gamma(i-l+1) \Gamma^{2}(l+1)(n+l+p+1)}, \\
& \text { where } \delta_{i, v}= \begin{cases}0, & \text { if } j \neq v, \\
1, & \text { if } j=v .\end{cases}
\end{aligned}
$$

Therefore (28) yields

$$
\begin{aligned}
I_{x}^{p} \mathfrak{Q}_{\mu}(x) \mathfrak{Q}_{\nu}(y) \\
=\sum_{n=0}^{\mu} \frac{(-1)^{\mu+n} \Gamma(\mu+n+1)}{\Gamma(\mu-n+1) \Gamma^{2}(n+1) \Gamma(n+p+1)} \\
\quad \cdot \sum_{i=0}^{m} \sum_{j=0}^{m} \mathscr{E}_{i, j, v} \mathfrak{Q}_{i}(x) \mathbb{R}_{j}(y)
\end{aligned}
$$




$$
\begin{aligned}
& =\sum_{i=0}^{m} \sum_{j=0}^{m} \sum_{n=0}^{\mu} \frac{(-1)^{\mu+n} \Gamma(\mu+n+1) \mathscr{E}_{i, j, \nu}}{\Gamma(\mu-n+1) \Gamma(n+1) \Gamma(n+p+1)} \mathfrak{Q}_{i}(x) \\
& \cdot \mathfrak{Q}_{j}(y)=\sum_{i=0}^{m} \sum_{j=0}^{m} \mathscr{E}_{i, j, \mu, \nu, n} \mathfrak{\Omega}_{i}(x) \mathfrak{\Omega}_{j}(y),
\end{aligned}
$$

where

$$
\mathscr{E}_{i, j, \mu, v, n}=\sum_{n=0}^{\mu} \frac{(-1)^{\mu+n} \Gamma(\mu+n+1) \mathscr{E}_{i, j, v}}{\Gamma(\mu-n+1) \Gamma(n+1) \Gamma(n+p+1)} .
$$

We use these notations for easiness of $u=K i+j+1, v=$ $K \mu+\nu+1, ¥_{v, u, n}=\mathscr{E}_{i, j, v, \mu, n}$ for $i, j, \mu, v=0,1,2,3, \ldots, m$. Thus, we received the required result

Theorem 7. The fractional order derivative of $\Upsilon_{K^{2}}(x, y)$ can be written as

$$
{ }^{c} D_{x}^{p}\left[\Upsilon_{K^{2}}(x, y)\right] \simeq \mathbf{W}_{K^{2} \times K^{2}}^{(p, x)} \Upsilon_{K^{2}}(x, y)
$$

$$
\mho_{i, j, \mu, v, n}=\sum_{l=[p]}^{v} \delta_{j, v}(2 i+1) \sum_{n=0}^{i} \frac{(-1)^{i+n+l+\mu} \Gamma(i+n+1) \Gamma(\mu+l+1)}{\Gamma(i-n+1) \Gamma^{2}(n+1) \Gamma(\mu-n+1) \Gamma(l+1) \Gamma(l-p+1)(n+l-p+1)}
$$

with $\mho_{i, j, \mu, v, n}=0$, if $\mu<p$.

where $v=K i+j+1, u=K \mu+v+1, ¥_{v, u, n}=$ $\Omega_{i, j, \mu, v, n}$ for $i, j, \mu, v=0,1,2, \ldots, m$,
Proof. Take $p$ the order fractional derivative of $\mathfrak{\Omega}_{n}(x, y)$ corresponding to $x$ as

$$
\begin{aligned}
& { }^{c} D_{x}^{p} \mathfrak{Q}_{k}(x, y)={ }^{c} D_{x}^{p} \mathfrak{Q}_{\mu}(x) \mathfrak{Q}_{v}(y) \\
& =\sum_{n=0}^{\mu} \frac{(-1)^{\mu+n} \Gamma(\mu+n+1)}{\Gamma(\mu-p+1) \Gamma^{2}(n+1)}{ }^{c} D_{x}^{p} x^{n} \boldsymbol{Q}_{v}(y) \\
& =\sum_{l=[p]}^{v} \frac{(-1)^{\mu+n} \Gamma(\mu+n+1)}{\Gamma(\mu-n+1) \Gamma^{2}(n+1) \Gamma(1+n-p)} x^{n-p} \mathfrak{Q}_{v}(y) .
\end{aligned}
$$

Approximate $x^{n-p} \mathfrak{Q}_{\nu}(y)$ in terms of shifted Legendre polynomials as

$$
x^{n-p} \mathbf{Q}_{\nu}(y) \approx \sum_{i=0}^{m} \sum_{j=0}^{m} \mathbf{D}_{i, j} \mathfrak{\Omega}_{i}(x) \mathbf{\Omega}_{j}(y) .
$$

Applying orthogonality condition, we may write

$$
\begin{aligned}
& \mathbf{D}_{i, j} \\
& = \begin{cases}0, & \text { if } j \neq v, \\
(2 i+1) \sum_{n=0}^{i} \frac{(-1)^{i+n} \Gamma(i+n+1)}{\Gamma(i-n+1) \Gamma^{2}(n+1)(n+l-p+1)}, & \text { if } j=v .\end{cases}
\end{aligned}
$$

Therefore, we get

$$
\begin{aligned}
& { }^{c} D_{x}^{p} \mathbf{Q}_{k}(x, y)=\sum_{n=[p]}^{\mu} \frac{(-1)^{\mu+n} \Gamma(\mu+n+1)}{\Gamma(\mu-n+1) \Gamma(n+1)^{2} \Gamma(n-p+1)} \\
& \cdot \sum_{i=0}^{m} \sum_{j=0}^{m} \mathbf{D}_{i, j} \mathfrak{Q}_{i}(x) \mathbf{Q}_{j}(y) \\
& =\sum_{i=0}^{m} \sum_{j=0}^{m} \sum_{n=[p]}^{\mu} \frac{(-1)^{\mu+n} \Gamma(\mu+n+1)}{\Gamma(\mu-n+1) \Gamma(n+1)^{2} \Gamma(n-p+1)} \mathbf{D}_{i, j} \mathfrak{Q}_{i}(x) \\
& \cdot \mathbf{Q}_{j}(y)=\sum_{i=0}^{m} \sum_{j=0}^{m} \mho_{i, j, n, \mu, \nu} \mathfrak{Q}_{i}(x) \mathfrak{Q}_{j}(y), \quad \mu=[p], \ldots, K,
\end{aligned}
$$

where

$$
\begin{aligned}
& \mho_{i, j, n, \mu, \nu} \\
& \quad=\sum_{n=[p]}^{\mu} \frac{(-1)^{\mu+n} \Gamma(\mu+n+1)}{\Gamma(\mu-n+1) \Gamma(n+1)^{2} \Gamma(n-p+1)} \mathbf{D}_{i, j} .
\end{aligned}
$$

Denoting $u=K i+j+1, v=K \mu+v+1, ¥_{v, u, n}=\mho_{i, j, v, \mu, n}$, such that $i, j, \mu, \nu=0,1,2,3, \ldots, m$, for easiness, we get the required.

Theorem 8. The fractional derivative with order $p$ of $\Upsilon_{K^{2}}(x, y)$ with respect to $y$ can be written as

$$
{ }^{c} D_{y}^{\alpha}\left[\Upsilon_{K^{2}}(x, y)\right] \simeq \mathbf{V}_{K^{2} \times K^{2}}^{(p, y)} ¥_{K^{2}}(x, y),
$$


such that $\mathbf{V}_{K^{2} \times K^{2}}^{(p, y)}$ is the operational matrix given as $\mathbf{V}_{K^{2} \times K^{2}}^{(p, y)}$

$$
=\left(\begin{array}{cccccc}
¥_{1,1, n} & ¥_{1,2, n} & \cdots & ¥_{1, u, n} & \cdots & ¥_{1, K^{2}, n} \\
¥_{2,1, n} & ¥_{2,2, n} & \cdots & ¥_{2, u, n} & \cdots & ¥_{2, K^{2}, n} \\
\vdots & \vdots & \vdots & \vdots & \vdots & \vdots \\
¥_{v, 1, n} & ¥_{v, 2, n} & \cdots & ¥_{v, u, n} & \cdots & ¥_{v, K^{2}, n} \\
\vdots & \vdots & \vdots & \vdots & \vdots & \vdots \\
¥_{K^{2}, 1, n} & ¥_{K^{2}, 2, n} & \cdots & ¥_{K^{2}, u, n} & \cdots & ¥_{K^{2}, K^{2}, n}
\end{array}\right),
$$

$$
\mho_{i, j, \mu, \nu, n}=\sum_{l=[p]}^{\nu} \delta_{i, \mu} \sum_{l=0}^{j} \frac{(-1)^{j+l+v+n} \Gamma(j+l+1) \Gamma(\nu+n+1)}{\Gamma(j-l+1) \Gamma^{2}(l+1) \Gamma(\nu-n+1) \Gamma(n+1) \Gamma(n-p+1)(n+l-p+1)},
$$

where $\mho_{i, j, \mu, v, n}=0$, if $\nu<p$.
Proof. To prove this theorem it can be easily followed from the proof of Theorem 7 .

Theorem 9. The fractional derivative of $\Upsilon_{K^{2}}(x, y)$ with respect to $x, y$ is provided as

$$
{ }^{c} D_{x y}^{p}\left[\Upsilon_{K^{2}}(x, y)\right] \simeq \mathbf{Z}_{K^{2} \times K^{2}}^{(p, x, y)} \Upsilon_{K^{2}}(x, y),
$$

where $\mathbf{Z}_{K^{2} \times K^{2}}^{(p, x, y)}$ represents the operational matrix of fractional derivative given as
$u=K i+j+1, \quad v=K \mu+v+1, ¥_{v, u, n}=\mho_{i, j, \mu, v, n}$ for $i, j, \mu, v=$ $0,1,2, \ldots, m$ and 
We approximate $x^{(n-p / 2)} y^{(l-p / 2)}$ in terms of shifted Legendre polynomials as

$$
x^{(n-p / 2)} y^{(l-p / 2)} \approx \sum_{i=0}^{m} \sum_{j=0}^{m} D_{(i, j, n, l)} \mathfrak{Q}_{i}(x) \mathfrak{Q}_{j}(y),
$$

such that

$$
\begin{aligned}
& D_{(i, j, n, l)}=(2 i+1)(2 j+1) \int_{0}^{1} \int_{0}^{1} \mathfrak{Q}_{\mu}(x) \mathfrak{Q}_{\nu}(y) \mathfrak{R}_{i}(x) \\
& \cdot \mathfrak{Q}_{j}(y) x^{n-p / 2} y^{l-p / 2} d x d y .
\end{aligned}
$$

Thanks to the orthogonality conditions and convolution theorem of Laplace transform, evaluating the integrals (49), we get

$$
D_{(i, j, n, l)}=\delta_{i, j, \mu, \nu}(2 i+1)(2 j+1) \times \sum_{n=0}^{i} \sum_{l=0}^{j} \frac{(-1)^{i+j+n+l} \Gamma(j+l+1) \Gamma(i+n+1)}{\Gamma(j-l+1) \Gamma(i-n+1) \Gamma^{2}(n+1) \Gamma^{2}(l+1)(n+l-p / 2+1)^{2}},
$$

where

$$
\delta_{i, j, \mu, \nu}= \begin{cases}1, & \text { if } i=\mu, j=v, \\ 0, & \text { if } i \neq \mu, j \neq v .\end{cases}
$$

Plugging (48) in (47), we may write

$$
\begin{aligned}
{ }^{c} D_{x y}^{p} \mathfrak{Q}_{k}(x, y) & =\sum_{n=[p / 2]}^{\mu} \sum_{l=[p / 2]}^{\nu} \frac{(-1)^{\mu+n} \Gamma(\mu+n+1)}{\Gamma(\mu-n+1) \Gamma(n+1)} \frac{(-1)^{\nu+l} \Gamma(\nu+l+1)}{\Gamma(\nu-l+1) \Gamma(l+1)} \sum_{i=0}^{m} \sum_{j=0}^{m} D_{(i, j, n, l)} \mathfrak{Q}_{i}(x) \mathfrak{Q}_{j}(y) \\
& =\sum_{i=0}^{m} \sum_{j=0}^{m} \sum_{n=[p / 2]}^{a} \sum_{l=[p / 2]}^{\nu} \frac{(-1)^{\mu+n} \Gamma(\mu+n+1)}{\Gamma(\mu-n+1) \Gamma(n+1)} \frac{(-1)^{\nu+l} \Gamma(\nu+l+1)}{\Gamma(\nu-l+1) \Gamma(l+1)} D_{(i, j, n, l)} \mathfrak{Q}_{i}(x) \mathfrak{Q}_{j}(y) .
\end{aligned}
$$

Representing $u=K \mu=v+1, v=K i+j+1$, and $¥_{v, u, n}=$ $\mho_{i, j, \mu, v, n, l}$ for $i, j, \mu, \nu=0,1,2, \ldots, m$, we obtain the required results.

\section{Solutions of the Coupled Systems of Equations}

Thanks to the operational matrices established in previous section, we now in position to obtain numerical solutions of the proposed coupled system (1) of FPDEs.

With the help of operational matrices, we consider the approximations as

$$
\begin{aligned}
& { }^{c} D_{x}^{p} U(x, y)=\mathbf{A}_{K^{2}} \Upsilon_{K^{2}}(x, y), \\
& { }^{c} D_{x}^{p} V(x, y)=\mathbf{B}_{K^{2}} \Upsilon_{K^{2}}(x, y) .
\end{aligned}
$$

In view of Theorem 4, we can write

$$
\begin{aligned}
& U(x, y)-\sum_{i=0}^{n} e_{i} x^{i}=\mathbf{A}_{K^{2}} \mathbf{H}_{K^{2} \times K^{2}}^{(p, x)} \Upsilon_{K^{2}}(x, y), \\
& V(x, y)-\sum_{i=0}^{n} b_{i} x^{i}=\mathbf{B}_{K^{2}} \mathbf{H}_{K^{2} \times K^{2}}^{(p, x)} \Upsilon_{K^{2}}(x, y) .
\end{aligned}
$$

Applying initial conditions, we get

$$
\begin{aligned}
& U(x, y)=\mathbf{A}_{K^{2}} \mathbf{H}_{K^{2} \times K^{2}}^{(p, x)} \Upsilon_{K^{2}}(x, y)+\mathbf{X}_{K^{2}}^{1} \Upsilon_{K^{2}}(x, y), \\
& V(x, y)=\mathbf{B}_{K^{2}} \mathbf{H}_{K^{2} \times K^{2}}^{(p, x)} \Upsilon_{K^{2}}(x, y)+\mathbf{X}_{K^{2}}^{2} \Upsilon_{K^{2}}(x, y),
\end{aligned}
$$

where $\mathbf{X}_{K^{2}}^{1} \Upsilon_{K^{2}}(x, y)=\sum_{i=0}^{n} \theta_{i}(y) x^{i}$ and $\mathbf{X}_{K^{2}}^{2} \Upsilon_{K^{2}}(x, y)=$ $\sum_{i=0}^{n} \psi_{i}(y) x^{i}$. In simple notation, we may write

$$
\begin{aligned}
& \mathbf{A}_{K^{2}} \mathbf{H}_{K^{2} \times K^{2}}^{(p, x)}+\mathbf{X}_{K^{2}}^{1}=\widehat{\mathbf{A}_{K^{2}}}, \\
& \mathbf{B}_{K^{2}} \mathbf{H}_{K^{2} \times K^{2}}^{(p, x)}+\mathbf{X}_{K^{2}}^{2}=\widehat{\mathbf{B}_{K^{2}}} .
\end{aligned}
$$

Therefore (55) yields

$$
\begin{aligned}
& U(x, y)=\widehat{\mathbf{A}_{K^{2}}} \Upsilon_{K^{2}}(x, y), \\
& V(x, y)=\widehat{\mathbf{B}_{K^{2}}} \Upsilon_{K^{2}}(x, y) .
\end{aligned}
$$

Thanks to (57), the other terms of system (1) can be approximated as

$$
\begin{aligned}
& { }^{c} D_{y}^{q_{1}} U(x, y)=\widehat{\mathbf{A}_{K^{2}}} \mathbf{V}_{K^{2} \times K^{2}}^{\left(q_{1}, y\right)} \Upsilon_{K^{2}}(x, y), \\
& { }^{c} D_{y}^{q_{2}} V(x, y)=\widehat{\mathbf{B}_{K^{2}}} \mathbf{V}_{K^{2} \times K^{2}}^{\left(q_{2}, y\right)} \Upsilon_{K^{2}}(x, y),
\end{aligned}
$$




$$
\begin{aligned}
{ }^{c} D_{x y}^{p_{1}} V(x, y) & =\widehat{\mathbf{B}_{K^{2}}} \mathbf{Z}_{K^{2} \times K^{2}}^{\left(p_{1}, x, y\right)} \Upsilon_{K^{2}}(x, y), \\
{ }^{c} D_{x y}^{p_{2}} U(x, y) & =\widehat{\mathbf{A}_{K^{2}}} \mathbf{Z}_{K^{2} \times K^{2}}^{\left(p_{2}, x, y\right)} \\
\theta(x, y) & =\mathbf{X}_{K^{2}}^{3} \Upsilon_{K^{2}}(x, y) \\
\psi(x, y) & =\mathbf{X}_{K^{2}}^{4} \Upsilon_{K^{2}}(x, y) .
\end{aligned}
$$

Putting in (1), we get

$$
\begin{aligned}
& \mathbf{A}_{K^{2}} \Upsilon_{K^{2}}(x, y)+\omega_{1} \widehat{\mathbf{B}_{K^{2}}} \mathbf{Z}_{K^{2} \times K^{2}}^{\left(p_{1}, x, y\right)} \Upsilon_{K^{2}}(x, y) \\
& +\omega_{2} \widehat{\mathbf{A}_{K^{2}}} \mathbf{V}_{K^{2} \times K^{2}}^{\left(q_{1}, y\right)} \Upsilon_{K^{2}}(x, y)=\mathbf{X}_{K^{2}}^{3} \Upsilon_{K^{2}}(x, y), \\
& \mathbf{B}_{K^{2}} \Upsilon_{K^{2}}(x, y)+\rho_{1} \widehat{\mathbf{A}_{K^{2}}} \mathbf{Z}_{K^{2} \times K^{2}}^{\left(p_{2}, x, y\right)} \Upsilon_{K^{2}}(x, y) \\
& \quad+\rho_{2} \widehat{\mathbf{B}_{K^{2}}} \mathbf{V}_{K^{2} \times K^{2}}^{\left(q_{2}, y\right)} \Upsilon_{K^{2}}(x, y)=\mathbf{X}_{K^{2}}^{4} \Upsilon_{K^{2}}(x, y) .
\end{aligned}
$$

Now we write matrix form of system (59) as

$$
\begin{gathered}
{\left[\begin{array}{c}
\mathbf{A}_{K^{2}} \Upsilon_{K^{2}}(x, y) \\
\mathbf{B}_{K^{2}} \Upsilon_{K^{2}}(x, y)
\end{array}\right]+\left[\begin{array}{c}
\omega_{1} \widehat{\mathbf{B}_{K^{2}}} \mathbf{Z}_{K^{2} \times K^{2}}^{\left(p_{1}, x, y\right)} \Upsilon_{K^{2}}(x, y) \\
\rho_{1} \widehat{\mathbf{A}_{K^{2}}} \mathbf{Z}_{K^{2} \times K^{2}}^{\left(p_{2}, x, y\right)} \Upsilon_{K^{2}}(x, y)
\end{array}\right]} \\
+\left[\begin{array}{l}
\omega_{2} \widehat{\mathbf{A}_{K^{2}}} \mathbf{V}_{K^{2} \times K^{2}}^{\left(\gamma_{2}, y\right)} \Upsilon_{K^{2}}(x, y) \\
\rho_{2} \widehat{\mathbf{B}_{K^{2}}} \mathbf{V}_{K^{2} \times K^{2}}^{\left(\rho_{2}, y\right)} \Upsilon_{K^{2}}(x, y)
\end{array}\right] \\
-\left[\begin{array}{l}
\mathbf{X}_{K^{2}}^{3} \Upsilon_{K^{2}}(x, y) \\
\mathbf{X}_{K^{2}}^{4} \Upsilon_{K^{2}}(x, y)
\end{array}\right]=\mathbf{O},
\end{gathered}
$$

which further gives

$$
\begin{aligned}
& {\left[\begin{array}{ll}
\mathbf{A}_{K^{2}} & \mathbf{B}_{K^{2}}
\end{array}\right] \widehat{\mathbf{E}}} \\
& +\left[\begin{array}{ll}
\widehat{\mathbf{A}_{K^{2}}} & \widehat{\mathbf{B}_{K^{2}}}
\end{array}\right]\left[\begin{array}{cc}
\mathbf{O}_{K^{2} \times K^{2}} & \rho_{1} \mathbf{Z}_{K^{2} \times K^{2}}^{\left(p_{2}, x, y\right)} \\
\omega_{1} \mathbf{Z}_{K^{2} \times K^{2}}^{\left(p_{1}, x, y\right)} & \mathbf{O}_{K^{2} \times K^{2}}
\end{array}\right] \widehat{\mathbf{E}} \\
& +\left[\begin{array}{ll}
\widehat{\mathbf{A}_{K^{2}}} & \widehat{\mathbf{B}_{K^{2}}}
\end{array}\right]\left[\begin{array}{cc}
\omega_{2} \mathbf{V}_{K^{2} \times K^{2}}^{\left(q_{1}, y\right)} & \mathbf{O}_{K^{2} \times K^{2}} \\
\mathbf{O}_{K^{2} \times K^{2}} & \rho_{2} \mathbf{V}_{K^{2} \times K^{2}}^{\left(q_{2}, y\right)}
\end{array}\right] \widehat{\mathbf{E}} \\
& -\left[\begin{array}{ll}
\mathbf{X}_{K^{2}}^{3} & \mathbf{X}_{K^{2}}^{4}
\end{array}\right] \widehat{\mathbf{E}}=\mathbf{O} \\
& {\left[\begin{array}{ll}
\mathbf{A}_{K^{2}} & \mathbf{B}_{K^{2}}
\end{array}\right]+\left[\begin{array}{ll}
\widehat{\mathbf{A}_{K^{2}}} & \widehat{\mathbf{B}_{K^{2}}}
\end{array}\right]\left[\begin{array}{cc}
\omega_{2} \mathbf{V}_{K^{2} \times K^{2}}^{\left(q_{1}, y\right)} & \rho_{1} \mathbf{Z}_{K^{2} \times K^{2}}^{\left(p_{2}, x, y\right)} \\
\omega_{1} \mathbf{Z}_{K^{2} \times K^{2}}^{\left(p_{1}, x, y\right)} & \rho_{2} \mathbf{V}_{K^{2} \times K^{2}}^{\left(q_{2}, y\right)}
\end{array}\right]} \\
& -\left[\begin{array}{ll}
\mathbf{X}_{K^{2}}^{3} & \mathbf{X}_{K^{2}}^{4}
\end{array}\right]=\mathbf{O},
\end{aligned}
$$

where

$$
\widehat{\mathbf{E}}=\left[\begin{array}{cc}
\Upsilon_{K^{2}}(x, y) & \mathbf{O}_{K^{2}} \\
\mathbf{O}_{K^{2}} & \Upsilon_{K^{2}}(x, y)
\end{array}\right] .
$$

$\mathbf{O}_{K^{2}}$ is a column null matrix and other null matrices are $\mathbf{O}_{K^{2} \times K^{2}}$ and $\mathbf{O}$.
Simplifying (61), we get

$$
\begin{gathered}
{\left[\begin{array}{ll}
\mathbf{A}_{K^{2}} & \mathbf{B}_{K^{2}}
\end{array}\right]-\left[\begin{array}{ll}
\widehat{\mathbf{A}_{K^{2}}} & \widehat{\mathbf{B}_{K^{2}}}
\end{array}\right]\left[\begin{array}{cc}
\omega_{2} \mathbf{V}_{K^{2} \times K^{2}}^{\left(q_{1}, y\right)} & \rho_{1} \mathbf{Z}_{K^{2} \times K^{2}}^{\left(p_{2}, x, y\right)} \\
\omega_{1} \mathbf{Z}_{K^{2} \times K^{2}}^{\left(p_{1}, x, y\right)} & \rho_{2} \mathbf{V}_{K^{2} \times K^{2}}^{\left(q_{2}, y\right)}
\end{array}\right]} \\
-\left[\begin{array}{ll}
\mathbf{X}_{K^{2}}^{3} & \mathbf{X}_{K^{2}}^{4}
\end{array}\right]=\mathbf{O} .
\end{gathered}
$$

Therefore by using $\widehat{\mathbf{A}_{K^{2}}}$ and $\widehat{\mathbf{B}_{K^{2}}}$, the aforesaid equation can be written as

$$
\begin{aligned}
& {\left[\begin{array}{ll}
\mathbf{A}_{K^{2}} & \mathbf{B}_{K^{2}}
\end{array}\right]-\widehat{\mathbf{P}}\left[\begin{array}{ll}
\mathbf{A}_{K^{2}} & \mathbf{B}_{K^{2}}
\end{array}\right]-\left[\begin{array}{ll}
\mathbf{X}_{K^{2}}^{1} & \mathbf{X}_{K^{2}}^{2}
\end{array}\right] \widehat{\mathbf{P}}} \\
& \quad-\left[\begin{array}{ll}
\mathbf{F}_{1} & \mathbf{F}_{2}
\end{array}\right]=\mathbf{O},
\end{aligned}
$$

where

$$
\begin{aligned}
\widehat{\mathbf{P}} & =\left[\begin{array}{cc}
\omega_{2} \mathbf{H}_{K^{2} \times K^{2}}^{(p, x)} \mathbf{V}_{K^{2} \times K^{2}}^{\left(q_{1}, y\right)} & \rho_{1} \mathbf{H}_{K^{2} \times K^{2}}^{(p, x)} \mathbf{Z}_{K^{2} \times K^{2}}^{\left(p_{2}, x, y\right)} \\
\omega_{2} \mathbf{H}_{K^{2} \times K^{2}}^{(p, x)} \mathbf{Z}_{K^{2} \times K^{2}}^{\left(p_{1}, x, y\right)} & \rho_{2} \mathbf{H}_{K^{2} \times K^{2}}^{(p, x)} \mathbf{V}_{K^{2} \times K^{2}}^{\left(q_{2}, y\right)}
\end{array}\right], \\
\mathbf{F}_{1} & =\omega_{2} \mathbf{X}_{K^{2}}^{1} \mathbf{V}_{K^{2} \times K^{2}}^{\left(q_{1}, y\right)}+\omega_{1} \mathbf{X}_{K^{2}}^{2} \mathbf{Z}_{K^{2} \times K^{2}}^{\left(p_{1}, x, y\right)}-\mathbf{X}_{K^{2}}^{3}, \\
\mathbf{F}_{2} & =\rho_{1} \mathbf{X}_{K^{2}}^{1} \mathbf{W}_{K^{2} \times K^{2}}^{\left(p_{2}, x\right)}+\rho_{2} \mathbf{X}_{K^{2}}^{2} \mathbf{V}_{K^{2} \times K^{2}}^{\left(q_{2}, y\right)}-\mathbf{X}_{K^{2}}^{4} .
\end{aligned}
$$

Hence (64) is Sylvester type matrix equation, which on solving for unknown matrix $\left[\begin{array}{ll}\mathbf{A}_{K^{2}} & \mathbf{B}_{K^{2}}\end{array}\right]$ and using its value in (57), we get the required numerical solution of the proposed problem.

Remark 10. Here, we remark that we have used a machine type DESKTOP-V8125H8 with processor intel(R) Core (TM) i5-3210M CPU@ $2.50 \mathrm{GHz}$ and installed memory (RAM) is $4 \mathrm{~GB}$ together with 64-bit operating system for computation and numerical simulations.

\section{Numerical Test Problems}

This section is concerning to the numerical test problems and their visualization.

Problem 1. Let the coupled system of FPDEs given as

$$
\begin{aligned}
& { }^{c} D_{x}^{1.8} U(x, y)-{ }^{c} D_{x y}^{2} V(x, y)-4^{c} D_{y}^{1.8} U(x, y) \\
& \quad=\theta(x, y), \\
& { }^{c} D_{x}^{1.8} V(x, y)-6{ }^{c} D_{x y}^{2} U(x, y)+3^{c} D_{y}^{1.8} V(x, y) \\
& \quad=\psi(x, y), \\
& U(0, y)=U^{\prime}(0, y)=0, \\
& V(0, y)=V^{\prime}(0, y)=0,
\end{aligned}
$$

such that the external functions $\theta(x, y)$ and $\phi(x, y)$ are given as

$$
\begin{aligned}
& \theta(x, y)=27 x^{2} y^{2} 9(y-1)(x-1)^{2}(7 x y-2 y-3) \\
& -4 x^{4} y^{3}(4+3 y)-0.016 x^{2.5} y^{4}(y-1)^{3}\left(125 x^{2}\right. \\
& \quad-175 x+56)
\end{aligned}
$$



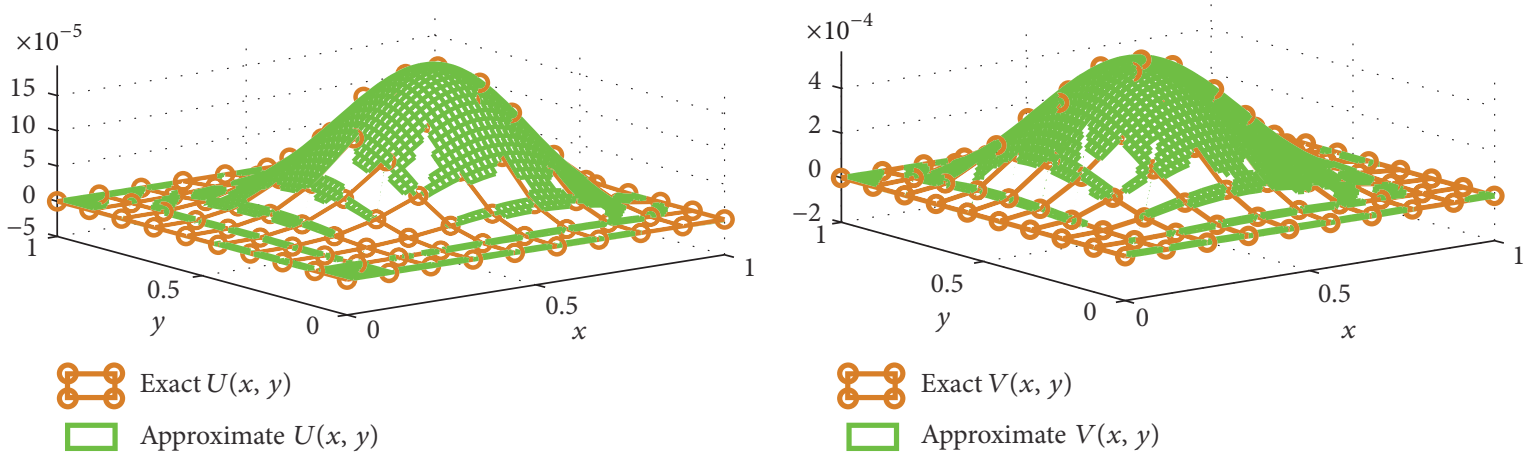

FIgURE 1: Evaluation of numerical and exact solutions $U(x, y), V(x, y)$ corresponding to scale level $K=10$ of Problem 1.
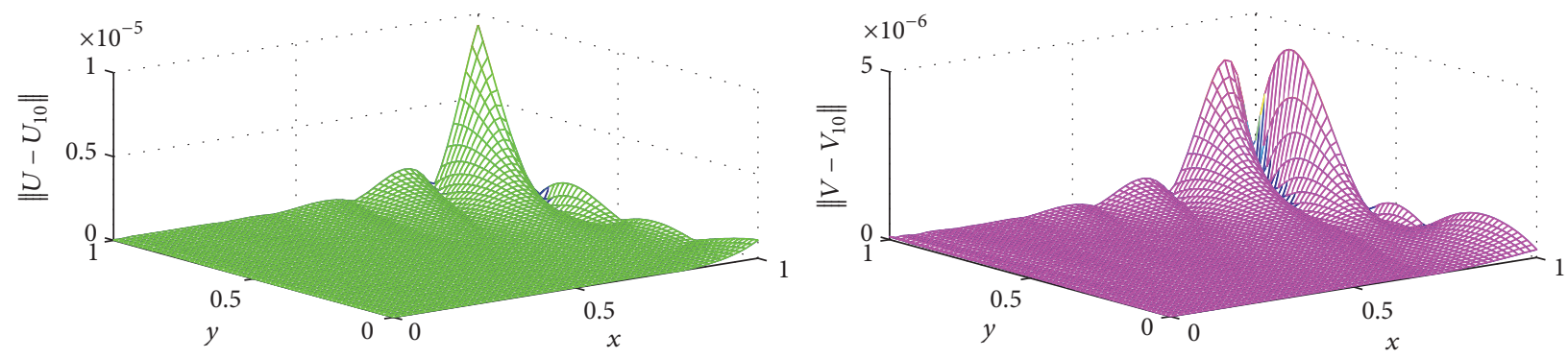

Figure 2: Absolute error in $U(x, y)$ and $V(x, y)$ using $K=10$ of Problem 1.

$$
\begin{aligned}
& \phi(x, y)=-36 x^{2} y^{3}(y-1)(x-1)^{3}[1 \\
& -2 x^{2}\left(\frac{y-1}{x-1}\right)^{2}-\frac{3 x^{2}(y-1)}{2(x-1)^{2}}+\frac{3(y-1)}{2 y} \\
& \left.\quad-\frac{x(y-1)}{x-1}-\frac{y-1}{4 x}-4 x\left(\frac{y-1}{x-1}\right)^{2}-3 x y \frac{y-1}{x-1}\right] \\
& -0.071 x^{1.2} y^{3}(y-1)^{2}\left(1250 x^{3}-2625 x^{2}+1680 x\right. \\
& -308) .
\end{aligned}
$$

The exact solution of the above system is

$$
\begin{aligned}
& U(x, y)=(x y(1-x))^{2}(1-y)^{3}, \\
& V(x, y)=x y(1-y)\left(x y-x^{2} y-x y^{2}\right)^{2} .
\end{aligned}
$$

Evaluate the approximate solution of Problem 1 with our proposed method. After applying the method to the given problem, we see from Figure 1 that the considered scheme provides close agreement between numerical and exact solution. The comparison between exact and approximate solution is shown in Figure 1, while the absolute error corresponding to scale level $K=10$ is provided in Figure 2. Further, to check the efficiency of the method, we have also computed absolute error at various scale level and different points of the spaces as given in Table 1.
Further in Table 2, we compute the CPU time for the computational of solutions for the test Problem 1.

Problem 2. To support the aforesaid established results, we consider the following problem:

$$
\begin{aligned}
& { }^{c} D_{x}^{1.8} U(x, y)-{ }^{c} D_{x y}^{2} V(x, y)+11^{c} D_{y}^{0.99} U(x, y) \\
& \quad=\theta(x, y), \\
& \quad=\psi(x, y), \\
& { }^{c} D_{x}^{1.8} V(x, y)+{ }^{c} D_{x y}^{2} U(x, y)-11^{c} D_{y}^{0.99} V(x, y) \\
& U^{\prime}(0, y)=y, \\
& V(0, y)=0, \\
& V^{\prime}(0, y)=\exp (y) .
\end{aligned}
$$

The external source functions are provided as

$$
\begin{aligned}
\theta(x, y)= & \frac{y}{x^{1.8}} E_{1,-0.8}(x)-\exp (y)+11.046 y^{0.1} \\
\psi(x, y)= & 4.59 \exp (y) x^{0.8}+\exp (x)^{x} \\
& -11 x y^{-0.99} E_{1,0.1}
\end{aligned}
$$

where the exact solution of the Problem 2 is $U(x, y)=$ $y \exp (x), V(x, y)=x \exp (y)$. We approximate the given 
TABLE 1: Absolute error at various values of $(x, y)$ for $K=10,12$ in $U(x, y)$ and $V(x, y)$ of Problem 1 .

\begin{tabular}{lcccc}
\hline$(x, y)$ & $\left\|U-U_{10}\right\|$ & $\left\|U-U_{12}\right\|$ & $\left\|V-V_{10}\right\|$ & $\left\|V-V_{12}\right\|$ \\
\hline$(0.1,0.1)$ & $1.707 \times 10^{-5}$ & $1.005 \times 10^{-8}$ & $1.3250 \times 10^{-6}$ & $8.006 \times 10^{-9}$ \\
$(0.1,0.5)$ & $1.956 \times 10^{-5}$ & $6.006 \times 10^{-8}$ & $1.596 \times 10^{-6}$ & $7.508 \times 10^{-9}$ \\
$(0.1,0.9)$ & $2.236 \times 10^{-5}$ & $5.803 \times 10^{-8}$ & $5.773 \times 10^{-6}$ & $6.009 \times 10^{-9}$ \\
$(0.5,0.1)$ & $1.873 \times 10^{-5}$ & $4.600 \times 10^{-8}$ & $7.858 \times 10^{-6}$ & $7.036 \times 10^{-9}$ \\
$(0.5,0.5)$ & $1.887 \times 10^{-5}$ & $3.008 \times 10^{-8}$ & $6.619 \times 10^{-6}$ & $5.005 \times 10^{-9}$ \\
$(0.5,0.9)$ & $2.092 \times 10^{-5}$ & $2.519 \times 10^{-8}$ & $5.363 \times 10^{-6}$ & $4.001 \times 10^{-9}$ \\
$(0.9,0.1)$ & $1.973 \times 10^{-5}$ & $2.363 \times 10^{-8}$ & $6.009 \times 10^{-6}$ & $2.506 \times 10^{-9}$ \\
$(0.9,0.5)$ & $2.907 \times 10^{-5}$ & $5.009 \times 10^{-8}$ & $5.000 \times 10^{-6}$ & $3.005 \times 10^{-9}$ \\
$(0.9,0.9)$ & $2.306 \times 10^{-5}$ & $6.000 \times 10^{-8}$ & & $2.500 \times 10^{-9}$ \\
\hline
\end{tabular}

TABle 2: Absolute error and CPU times at various values of $(x, y)$ for $K=12$ in $U(x, y)$ and $V(x, y)$ of Problem 1.

\begin{tabular}{lcccc}
\hline$(x, y)$ & CPU time & $\left\|U-U_{12}\right\|$ & CPU time & $\left\|V-V_{12}\right\|$ \\
\hline$(0.1,0.1)$ & $55.78 \mathrm{~s}$ & $1.005 \times 10^{-8}$ & $57.88 \mathrm{~s}$ & $8.006 \times 10^{-9}$ \\
$(0.1,0.5)$ & $56.71 \mathrm{~s}$ & $6.006 \times 10^{-8}$ & $57.98 \mathrm{~s}$ & $7.508 \times 10^{-9}$ \\
$(0.1,0.9)$ & $56.91 \mathrm{~s}$ & $5.803 \times 10^{-8}$ & $57.99 \mathrm{~s}$ & $6.009 \times 10^{-9}$ \\
$(0.5,0.1)$ & $56.98 \mathrm{~s}$ & $4.600 \times 10^{-8}$ & $58.70 \mathrm{~s}$ & $7.036 \times 10^{-9}$ \\
$(0.5,0.5)$ & $57.31 \mathrm{~s}$ & $3.008 \times 10^{-8}$ & $58.72 \mathrm{~s}$ & $5.005 \times 10^{-9}$ \\
$(0.5,0.9)$ & $57.62 \mathrm{~s}$ & $2.519 \times 10^{-8}$ & $58.88 \mathrm{~s}$ & $4.001 \times 10^{-9}$ \\
$(0.9,0.1)$ & $58.11 \mathrm{~s}$ & $2.363 \times 10^{-8}$ & $59.23 \mathrm{~s}$ & $2.506 \times 10^{-9}$ \\
$(0.9,0.5)$ & $59.41 \mathrm{~s}$ & $5.009 \times 10^{-8}$ & $59.38 \mathrm{~s}$ & $3.005 \times 10^{-9}$ \\
$(0.9,0.9)$ & $59.88 \mathrm{~s}$ & $6.000 \times 10^{-8}$ & $60.08 \mathrm{~s}$ & $2.500 \times 10^{-9}$ \\
\hline
\end{tabular}
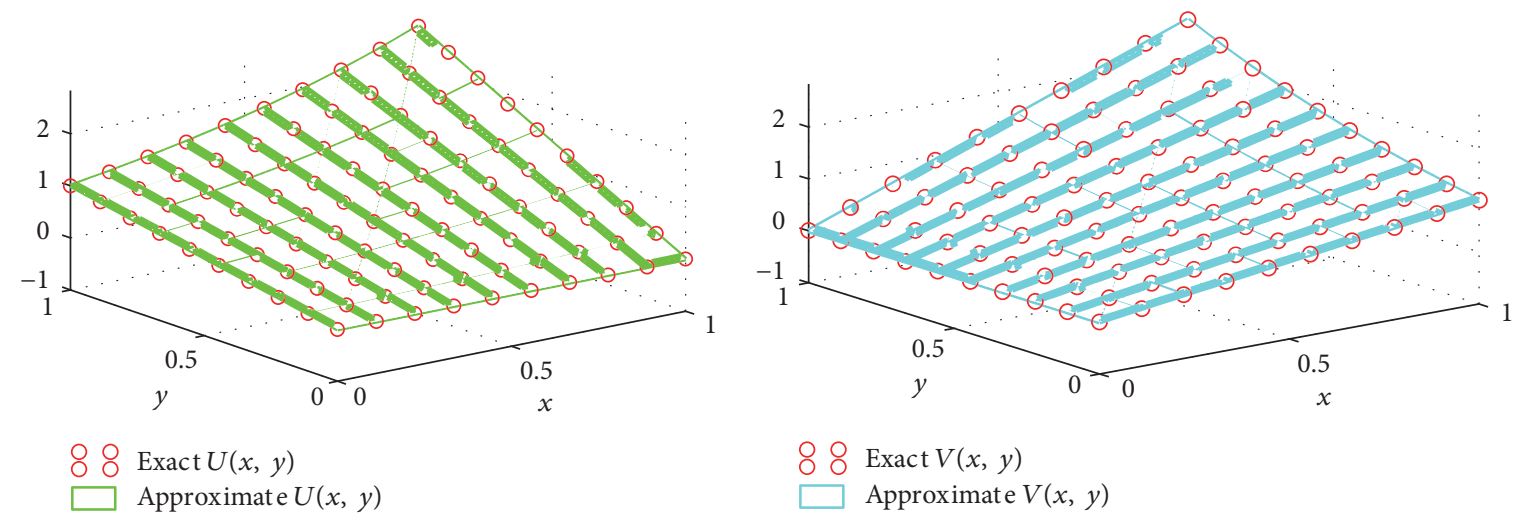

FIGURE 3: Evaluation of exact and numerical solutions $U(x, y), V(x, y)$ corresponding to scale level $K=10$ for test Problem 2.

test problem by using the proposed method. In Figure 3, we have shown the comparison between exact and approximate solutions at scale level $K=10$. Further, to verify the efficiency of the proposed method, also the absolute error at scale level $K=10$ in $U(x, y)$ and $V(x, y)$ has been shown in Figure 4. To demonstrate the maximum absolute error at different scale levels and various values of the space variables $x, y$ has been provided in Table 3. From Table 3, it is clear that the absolute error is below $10^{-8}$, which is a very small quantity. The absolute error may further be decreased by enlarging scale level. CPU time for test Problem 2 is provided in Table 4.
Problem 3. Consider another coupled system of FPDEs as

$$
\begin{aligned}
& { }^{c} D_{x}^{1.8} U(x, y)+{ }^{c} D_{x y}^{2} V(x, y)+4^{c} D_{y}^{0.8} U(x, y) \\
& \quad=f(x, y), \\
& \quad=g(x, y), \\
& { }^{c} D_{x}^{1.8} V(x, y)+9^{c} D_{x y}^{2} V(x, y)+3^{c} D_{y}^{0.8} V(x, y) \\
& U(0, y)=-y^{5},
\end{aligned}
$$



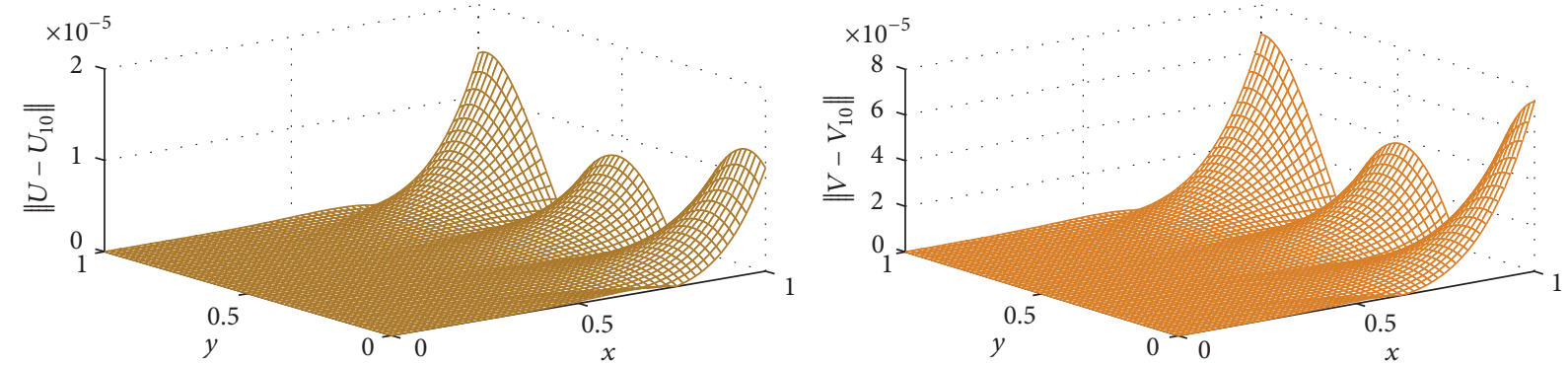

Figure 4: The maximum absolute error in $U(x, y)$ and $V(x, y)$ at $K=10$ of Problem 2.

TABle 3: Maximum absolute error at $K=10,12$ of $U(x, y)$ and $V(x, y)$ using various values of $(x, y)$ for Problem 2.

\begin{tabular}{lcccc}
\hline$(x, y)$ & $\left\|U-U_{10}\right\|$ & $\left\|U-U_{12}\right\|$ & $\left\|V-V_{10}\right\|$ & $\left\|V-V_{12}\right\|$ \\
\hline$(0.1,0.1)$ & $1.000 \times 10^{-5}$ & $2.005 \times 10^{-7}$ & $1.006 \times 10^{-5}$ & $7.908 \times 10^{-8}$ \\
$(0.1,0.5)$ & $7.000 \times 10^{-5}$ & $1.596 \times 10^{-7}$ & $2.000 \times 10^{-5}$ & $7.009 \times 10^{-8}$ \\
$(0.1,0.9)$ & $006 \times 10^{-5}$ & $1.003 \times 10^{-7}$ & $2.126 \times 10^{-5}$ & $5.019 \times 10^{-8}$ \\
$(0.5,0.1)$ & $6.003 \times 10^{-5}$ & $5.000 \times 10^{-7}$ & $2.451 \times 10^{-5}$ & $5.008 \times 10^{-8}$ \\
$(0.5,0.5)$ & $1.448 \times 10^{-5}$ & $4.000 \times 10^{-7}$ & $5.081 \times 10^{-5}$ & $4.052 \times 10^{-8}$ \\
$(0.5,0.9)$ & $2.500 \times 10^{-5}$ & $8.619 \times 10^{-7}$ & $6.050 \times 10^{-6}$ & $4.051 \times 10^{-8}$ \\
$(0.9,0.1)$ & $1.527 \times 10^{-5}$ & $7.063 \times 10^{-7}$ & $8.008 \times 10^{-6}$ & $3.094 \times 10^{-8}$ \\
$(0.9,0.5)$ & $2.5307 \times 10^{-6}$ & $4.129 \times 10^{-7}$ & $9.004 \times 10^{-6}$ & $2.096 \times 10^{-8}$ \\
$(0.9,0.9)$ & $1.706 \times 10^{-6}$ & $2.002 \times 10^{-7}$ & & $6.009 \times 10^{-8}$ \\
\hline
\end{tabular}

TABLE 4: Absolute error and CPU times at various values of $(x, y)$ for $K=12$ in $U(x, y)$ and $V(x, y)$ of Problem 2.

\begin{tabular}{lcccc}
\hline$(x, y)$ & CPU time & $\left\|U-U_{12}\right\|$ & CPU time & $\left\|V-V_{12}\right\|$ \\
\hline$(0.1,0.1)$ & $51.68 \mathrm{~s}$ & $2.005 \times 10^{-7}$ & $53.78 \mathrm{~s}$ & $7.908 \times 10^{-8}$ \\
$(0.1,0.5)$ & $51.71 \mathrm{~s}$ & $1.596 \times 10^{-7}$ & $53.90 \mathrm{~s}$ & $7.009 \times 10^{-8}$ \\
$(0.1,0.9)$ & $52.81 \mathrm{~s}$ & $1.003 \times 10^{-7}$ & $54.19 \mathrm{~s}$ & $5.019 \times 10^{-8}$ \\
$(0.5,0.1)$ & $52.98 \mathrm{~s}$ & $5.000 \times 10^{-7}$ & $55.20 \mathrm{~s}$ & $5.008 \times 10^{-8}$ \\
$(0.5,0.5)$ & $53.31 \mathrm{~s}$ & $4.000 \times 10^{-7}$ & $56.72 \mathrm{~s}$ & $4.052 \times 10^{-8}$ \\
$(0.5,0.9)$ & $53.62 \mathrm{~s}$ & $8.619 \times 10^{-7}$ & $56.38 \mathrm{~s}$ & $4.051 \times 10^{-8}$ \\
$(0.9,0.1)$ & $54.11 \mathrm{~s}$ & $7.063 \times 10^{-7}$ & $56.63 \mathrm{~s}$ & $3.094 \times 10^{-8}$ \\
$(0.9,0.5)$ & $55.41 \mathrm{~s}$ & $4.129 \times 10^{-7}$ & $56.88 \mathrm{~s}$ & $2.096 \times 10^{-8}$ \\
$(0.9,0.9)$ & $56.88 \mathrm{~s}$ & $2.002 \times 10^{-7}$ & $57.04 \mathrm{~s}$ & $6.009 \times 10^{-8}$ \\
\hline
\end{tabular}

$$
\begin{aligned}
& U^{\prime}(0, y)=0, \\
& V(0, y)=y^{5}, \\
& V^{\prime}(0, y)=0 .
\end{aligned}
$$

$$
\begin{aligned}
\psi(x, y)= & 5.508\left(y^{2}-x^{2}\right) x^{1.2}-1.836\left(x^{3}+y^{3}\right) x^{0.2} \\
& +54 x y(x-y)+82.62 y^{1.2}\left(x^{2}-y^{2}\right) \\
& -5.50\left(x^{3}+y^{3}\right) 6 y^{0.2} .
\end{aligned}
$$

While the external source term is given by

$$
\begin{aligned}
\theta(x, y)= & 4.54\left(x^{2}+y^{2}\right) x^{2.8}+9.18 x^{0.2} \\
& +2 x y(3 x-2)+58.08\left(x^{2}+y^{2}\right) x^{2.5} \\
& +10.632 x^{1.2}\left(x^{3}-y^{3}\right)
\end{aligned}
$$

The exact solution of Problem 3 at $p=2$ is

$$
\begin{aligned}
& U(x, y)=(x-y)\left[\left(x^{2}+y^{2}\right)^{2}+\left(x^{2}+y^{2}\right) x y\right], \\
& V(x, y)=(x+y)^{2}(y-x)\left(x^{2}+y^{2}-x y\right) .
\end{aligned}
$$



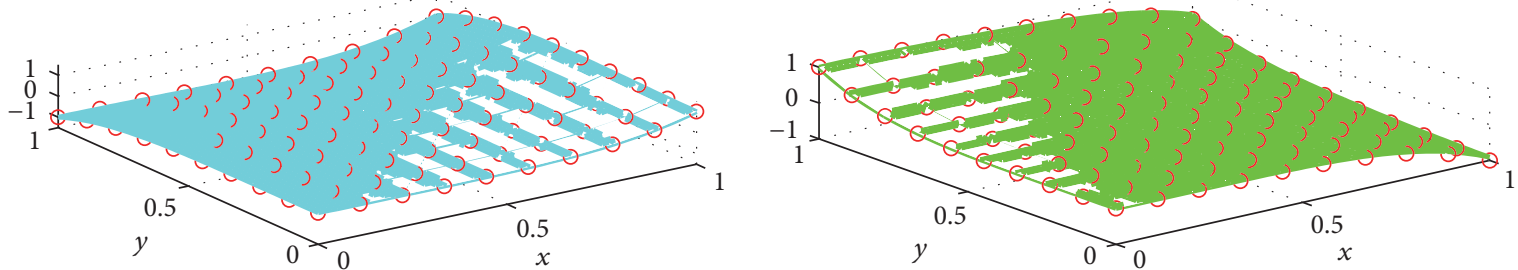

$\bigcirc 8 \operatorname{Exact} U(x, y)$

Approximate $U(x, y)$

$\bigcirc \bigcirc \operatorname{Exact} V(x, y)$

Approximate $V(x, y)$

Figure 5: Evaluation between exact and approximate solutions $U(x, y), V(x, y)$ for scale level $K=8$ of test Problem 3.
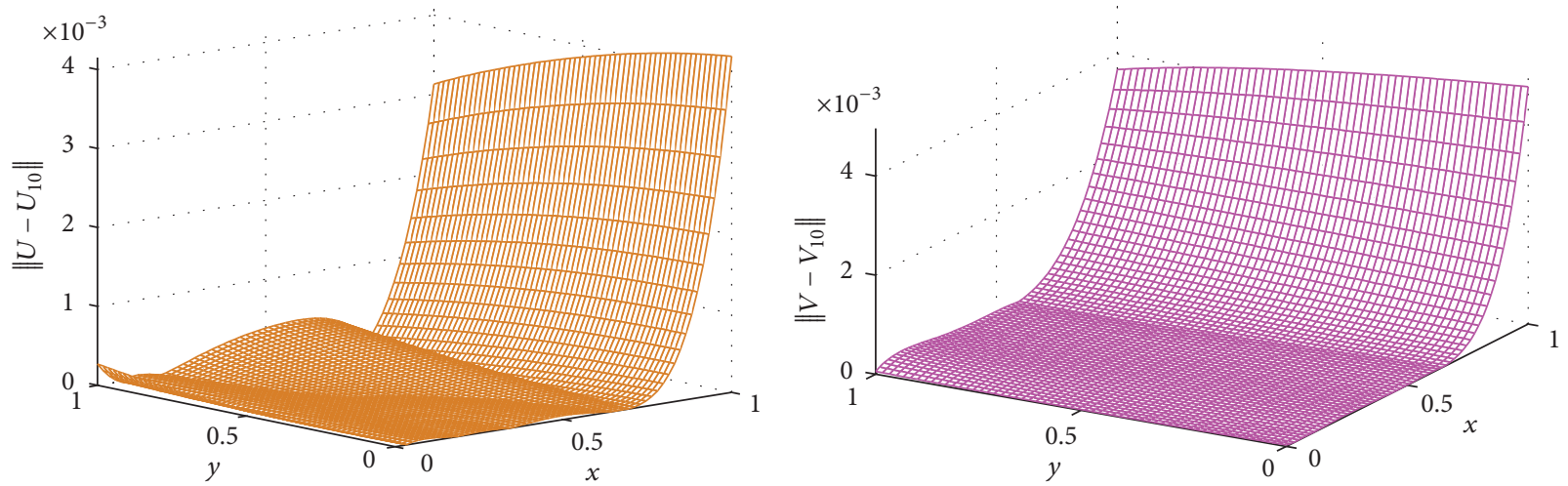

Figure 6: Maximum absolute error in $U(x, y)$ and $V(x, y)$ at $K=8$ for different values of $x, y$ for Problem 3.

TABle 5: Maximum absolute error in $U(x, y)$ and $V(x, y)$ of Problem 3 at $K=10,12$ corresponding to various values of $(x, y)$.

\begin{tabular}{lcccc}
\hline$(x, y)$ & $\left\|U-U_{10}\right\|$ & $\left\|U-U_{12}\right\|$ & $\left\|V-V_{10}\right\|$ & $\left\|V-V_{12}\right\|$ \\
\hline$(0.1,0.1)$ & $1.707 \times 10^{-6}$ & $4.325 \times 10^{-8}$ & $6.826 \times 10^{-6}$ & $1.000 \times 10^{-8}$ \\
$(0.1,0.5)$ & $2.956 \times 10^{-6}$ & $3.196 \times 10^{-8}$ & $3.638 \times 10^{-6}$ & $2.009 \times 10^{-8}$ \\
$(0.1,0.9)$ & $4.236 \times 10^{-6}$ & $2.703 \times 10^{-8}$ & $5.451 \times 10^{-6}$ & $2.900 \times 10^{-8}$ \\
$(0.5,0.1)$ & $8.873 \times 10^{-6}$ & $7.038 \times 10^{-8}$ & $4.781 \times 10^{-5}$ & $2.945 \times 10^{-8}$ \\
$(0.5,0.5)$ & $7.000 \times 10^{-5}$ & $8.058 \times 10^{-7}$ & $4.933 \times 10^{-5}$ & $1.520 \times 10^{-8}$ \\
$(0.5,0.9)$ & $8.002 \times 10^{-5}$ & $1.019 \times 10^{-7}$ & $4.565 \times 10^{-5}$ & $3.051 \times 10^{-8}$ \\
$(0.9,0.1)$ & $6.027 \times 10^{-5}$ & $3.063 \times 10^{-7}$ & $5.808 \times 10^{-5}$ & $3.194 \times 10^{-7}$ \\
$(0.9,0.5)$ & $9.007 \times 10^{-5}$ & $2.029 \times 10^{-7}$ & $2.746 \times 10^{-5}$ & $1.096 \times 10^{-7}$ \\
$(0.9,0.9)$ & $9.006 \times 10^{-5}$ & $2.008 \times 10^{-7}$ & & $3.209 \times 10^{-7}$ \\
\hline
\end{tabular}

Apply the considered method to the given Problem 3 corresponding to a scale level $K=8$. Plot the exact and approximate solution as in Figure 5. We see from Figure 5 that the adapted techniques work excellently and there is a close agreement between approximate and exact solutions. Further, we have computed maximum absolute error for Problem 3 in Table 5 at scale level $K=10,12$. The maximum absolute error corresponding to the scale level $K=8$ has been provided in Figure 6, which is below $10^{-3}$. The absolute error can be further decreased by enlarging scale level with various values of $x, y$.

CPU time for test Problem 3 is provided in Table 6.

\section{Conclusion}

In this article we have developed an efficient numerical technique by using shifted Legendre polynomials. A new operational matrix of mixed partial derivative $\mathbf{Z}_{K^{2} \times K^{2}}^{(p, x, y)}$ is introduced to solve a multiterm coupled system of FPDEs. We have also tested our method by some test problems. The comparison between the exact and approximate solutions of the problems has been provided. We observed that the maximum absolute error is very small even at very small scale level. This minimum value of the absolute error indicates that the method is highly accurate and can be applied to a large numbers of FPDEs. 
TABLE 6: Maximum absolute error and CPU times in $U(x, y)$ and $V(x, y)$ of Problem 3 at $K=12$ corresponding to various values of $(x, y)$.

\begin{tabular}{lcccc}
\hline$(x, y)$ & CPU time & $\left\|U-U_{12}\right\|$ & CPU time & $\left\|V-V_{12}\right\|$ \\
\hline$(0.1,0.1)$ & $55.70 \mathrm{~s}$ & $4.325 \times 10^{-8}$ & $55.26 \mathrm{~s}$ & $1.000 \times 10^{-8}$ \\
$(0.1,0.5)$ & $55.95 \mathrm{~s}$ & $3.196 \times 10^{-8}$ & $55.38 \mathrm{~s}$ & $2.009 \times 10^{-8}$ \\
$(0.1,0.9)$ & $56.23 \mathrm{~s}$ & $2.703 \times 10^{-8}$ & $56.12 \mathrm{~s}$ & $2.900 \times 10^{-8}$ \\
$(0.5,0.1)$ & $56.87 \mathrm{~s}$ & $7.038 \times 10^{-8}$ & $57.45 \mathrm{~s}$ & $2.945 \times 10^{-8}$ \\
$(0.5,0.5)$ & $57.00 \mathrm{~s}$ & $8.058 \times 10^{-7}$ & $75.78 \mathrm{~s}$ & $1.520 \times 10^{-8}$ \\
$(0.5,0.9)$ & $57.02 \mathrm{~s}$ & $1.019 \times 10^{-7}$ & $57.93 \mathrm{~s}$ & $3.051 \times 10^{-8}$ \\
$(0.9,0.1)$ & $57.27 \mathrm{~s}$ & $3.063 \times 10^{-7}$ & $58.56 \mathrm{~s}$ & $3.194 \times 10^{-7}$ \\
$(0.9,0.5)$ & $58.00 \mathrm{~s}$ & $2.029 \times 10^{-7}$ & $59.80 \mathrm{~s}$ & $1.096 \times 10^{-7}$ \\
$(0.9,0.9)$ & $59.68 \mathrm{~s}$ & $2.008 \times 10^{-7}$ & $60.76 \mathrm{~s}$ & $3.209 \times 10^{-7}$ \\
\hline
\end{tabular}

\section{Conflicts of Interest}

The authors declare that no conflicts of interest exist regarding this manuscript.

\section{Authors' Contributions}

Both authors equally contributed to this paper and approved the final version.

\section{Acknowledgments}

This work has been supported by the National Natural Science Foundation of China (11571378).

\section{References}

[1] R. W. Ibrahim, "Solutions to systems of arbitrary-order differential equations in complex domains," Electronic Journal of Differential Equations, vol. 46, pp. 1-13, 2014.

[2] K. B. Oldham, "Fractional differential equations in electrochemistry," Advances in Engineering Software, vol. 41, no. 1, pp. 9-12, 2010.

[3] R. Hilfer, Applications of Fractional Calculus in Physics, World Scientific, Singapore, 2000.

[4] G. Richards and P. R. R. Sarma, "Reduced order models for induction motors with two rotor circuits," IEEE Transactions on Energy Conversion, vol. 9, no. 4, pp. 673-678, 1994.

[5] R. Katica, "Dynamics of multi-pendulum systems with fractional order creep elements," Journal of Theoretical and Applied Mechanics, pp. 483-509, 2008.

[6] K. Shah, A. Ali, and R. A. Khan, "Numerical solutions of fractional order system of Bagley-Torvik equation using operational matrices," Sindh University Research Journal, vol. 47, no. 4, pp. 757-762, 2015.

[7] R. Katica and S. Hedrih, "Fractional order hybrid system dynamics," Proceedings in Applied Mathematics and Mechanics, vol. 13, pp. 25-26, 2013.

[8] K. Hedrih, "The transversal creeping vibrations of a fractional derivative order constitutive relation of nonhomogeneous beam," Mathematical Problems in Engineering, Article ID 46236, 18 pages, 2006.

[9] Y. Hu, Y. Luo, and Z. Lu, "Analytical solution of the linear fractional differential equation by Adomian decomposition method," Journal of Computational and Applied Mathematics, vol. 215, no. 1, pp. 220-229, 2008.

[10] H. Jafari and S. Seifi, "Solving a system of nonlinear fractional partial differential equations using homotopy analysis method," Communications in Nonlinear Science and Numerical Simulation, vol. 14, no. 5, pp. 1962-1969, 2009.

[11] A. Saadatmandi and M. Dehghan, "Numerical solution of a mathematical model for capillary formation in tumor angiogenesis via the tau method," Communications in Numerical Methods in Engineering with Biomedical Applications, vol. 24, no. 11, pp. 1467-1474, 2008.

[12] R. Metzler and J. Klafter, "Boundary value problems for fractional diffusion equations," Physica A. Statistical Mechanics and its Applications, vol. 278, no. 1-2, pp. 107-125, 2000.

[13] R. Metzler and J. Klafter, "The random walk's guide to anomalous diffusion: a fractional dynamics approach," Physics Reports. A Review Section of Physics Letters, vol. 339, no. 1, 77 pages, 2000.

[14] F. Liu, V. Anh, and I. Turner, "Numerical solution of the space fractional Fokker-Planck equation," vol. 166, no. 1, pp. 209-219.

[15] R. Gorenflo, F. Mainardi, E. Scalas, and M. Raberto, "Fractional calculus and continuous-time finance III. The diffusion limit," Mathematical Finance, pp. 171-180, 2000.

[16] M. ur Rehman and R. A. Khan, "Existence and uniqueness of solutions for multi-point boundary value problems for fractional differential equations," Applied Mathematics Letters. An International Journal of Rapid Publication, vol. 23, no. 9, pp. 1038-1044, 2010.

[17] R. A. Khan and M. ur Rehman, "Existence of multiple positive solutions for a general system of fractional differential equations," Communications on Applied Nonlinear Analysis, vol. 18, no. 1, pp. 25-35, 2011.

[18] M. u. Rehman, R. A. Khan, and N. A. Asif, "Three point boundary value problems for nonlinear fractional differential equations," Acta Mathematica Scientia. Series B. English Edition, vol. 31, no. 4, pp. 1337-1346, 2011.

[19] K. Shah, H. Khalil, and R. A. Khan, "Investigation of positive solution to a coupled system of impulsive boundary value problems for nonlinear fractional order differential equations," Chaos, Solitons and Fractals, vol. 77, pp. 240-246, 2015.

[20] K. Shah and R. A. Khan, "Existence and uniqueness of positive solutions to a coupled system of nonlinear fractional order differential equations with anti periodic boundary conditions," Differential Equations and Applications, vol. 7, no. 2, pp. 245262, 2015.

[21] J. Sundnes, G. T. Lines, K. A. Mardal, and A. Tveito, "Multigrid block preconditioning for a coupled system of partial 
differential equations modeling the electrical activity in the heart," Computer Methods in Biomechanics and Biomedical Engineering, vol. 5, no. 6, pp. 397-409, 2002.

[22] J. Sundnes, G. T. Lines, and A. Tveito, "An operator splitting method for solving the bidomain equations coupled to a volume conductor model for the torso," Mathematical Biosciences, vol. 194, no. 2, pp. 233-248, 2005.

[23] W. Shen, Computer Simulation and Modeling of Physical and Biological Processes using Partial Differential Equations [Doctoral, thesis], University of Kentucky Doctoral Dissertations, 2007.

[24] A. A. Moghadam, I. Aksikas, S. Dubljevic, and J. F. Forbes, "LQ control of coupled hyperbolic PDEs and ODEs: Application to a CSTR-PFR system," in Proceedings of the 9th International Symposium on Dynamics and Control of Process Systems (DYCOPS 2010), Leuven, Belgium, 2010.

[25] I. Aksikas, A. Fuxman, J. F. Forbes, and J. J. Winkin, "LQ control design of a class of hyperbolic PDE systems: application to fixedbed reactor," Automatica. A Journal of IFAC, the International Federation of Automatic Control, vol. 45, no. 6, pp. 1542-1548, 2009.

[26] Y. A. Rossikhin and M. V. Shitikova, "Application of fractional calculus for dynamic problems of solid mechanics: novel trends and recent results," Applied Mechanics Reviews, vol. 63, no. 1, Article ID 010801, 2010.

[27] V. Parthiban and K. Balachandran, "Solutions of system of fractional partial differential equations," Applications and Applied Mathematics, vol. 8, no. 1, pp. 289-304, 2013.

[28] K. Fackeldey and R. Krause, "Multiscale coupling in function space-weak coupling between molecular dynamics and continuum mechanics," International Journal for Numerical Methods in Engineering, vol. 79, no. 12, pp. 1517-1535, 2009.

[29] R. M. Wald, "Construction of solutions of gravitational, electromagnetic, or other perturbation equations from solutions of decoupled equations," Physical Review Letters, vol. 41, no. 4, pp. 203-206, 1978.

[30] A. Yildiz and C. H. Tang, "Electromagnetic cavity resonances in accelerated systems," Physical Review, vol. 146, no. 4, pp. 947954, 1966.

[31] A. Arikoglu and I. Ozkol, "Solution of fractional differential equations by using differential transform method," Chaos, Solitons \& Fractals, vol. 34, no. 5, pp. 1473-1481, 2007.

[32] V. S. Erturk and S. Momani, "Solving systems of fractional differential equations using differential transform method," Journal of Computational and Applied Mathematics, vol. 215, no. 1, pp. 142-151, 2008.

[33] K. Maleknejad, M. Shahrezaee, and H. Khatami, "Numerical solution of integral equations system of the second kind by block-pulse functions," Applied Mathematics and Computation, vol. 166, no. 1, pp. 15-24, 2005.

[34] Y. Wang and Q. Fan, "The second kind Chebyshev wavelet method for solving fractional differential equations," Applied Mathematics and Computation, vol. 218, no. 17, pp. 8592-8601, 2012.

[35] M. ur Rehman and R. Ali Khan, "The Legendre wavelet method for solving fractional differential equations," Communications in Nonlinear Science and Numerical Simulation, vol. 16, no. 11, pp. 4163-4173, 2011.

[36] M. Razzaghi and S. Yousefi, "The Legendre wavelets operational matrix of integration," International Journal of Systems Science. Principles and Applications of Systems and Integration, vol. 32, no. 4, pp. 495-502, 2001.
[37] M. Yi and Y. Chen, "Haar wavelet operational matrix method for solving fractional partial differential equations," Computer Modeling in Engineering and Sciences, vol. 88, no. 3, pp. 229244, 2012.

[38] H. Khalil and R. A. Khan, "A new method based on Legendre polynomials for solutions of the fractional two-dimensional heat conduction equation," Computers and Mathematics with Applications, vol. 67, no. 10, pp. 1938-1953, 2014.

[39] H. Khalil and R. Ali Khan, "The use of Jacobi polynomials in the numerical solution of coupled system of fractional differential equations," International Journal of Computer Mathematics, vol. 92, no. 7, pp. 1452-1472, 2015.

[40] H. Khalil and R. A. Khan, "Numerical scheme for solution of coupled system of initial value fractional order Fredholm integro-differential equations with smooth solutions," Journal of Mathematical Extension, vol. 9, no. 2, 19 pages, 2015.

[41] R. A. Khan and H. Khalil, "A new method based on legendre polynomials for solution of system of fractional order partial differential equations," International Journal of Computer Mathematics, vol. 91, no. 12, pp. 2554-2567, 2014.

[42] C. E. Lawrence, "Partial Differential Equations," in ISBN 0-82180772-2, American Mathematical Society, 1998.

[43] K. Shah and R. A. Khan, "Upper and lower solutions to a coupled system of nonlinear fractional differential equations," Progress in Fractional Differentiation and Applications, vol. 1, pp. 31-39, 2016.

[44] H. Khalil, R. A. Khan, D. Baleanu, and S. H. Saker, "Approximate solution of linear and nonlinear fractional differential equations under $m$-point local and nonlocal boundary conditions," Advances in Difference Equations, 28 pages, 2016.

[45] I. Podlubny, Fractional Differential Equations, Academic Press, San Diego, Calif, USA, 1999.

[46] A. A. Kilbas, H. M. Srivastava, and J. J. Trujillo, Theory and Applications of Fractional Differential Equations, Elsevier Science, Amsterdam, The Netherlands, 2006.

[47] K. Shah, Multipoint boundary value problems for systems of fractional differential equations: existence theory and numerical simulations [Ph.D. thesis], University of Malakand, Pakistan, 2016.

[48] S. Nemati and Y. Ordokhani, "Legendre expansion methods for the numerical solution of nonlinear 2D Fredholm integral equations of the second kind," Journal of Applied Mathematics \& Informatics, vol. 31, no. 5-6, pp. 609-621, 2013.

[49] A. Saadatmandi and M. Dehghan, "A new operational matrix for solving fractional-order differential equations," Computers and Mathematics with Applications, vol. 59, no. 3, pp. 1326-1336, 2010. 


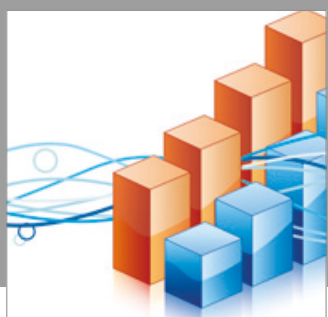

Advances in

Operations Research

vatersals

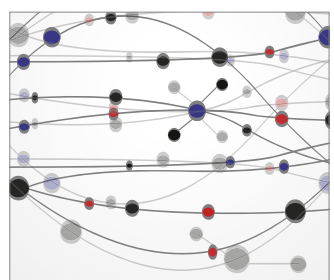

\section{The Scientific} World Journal
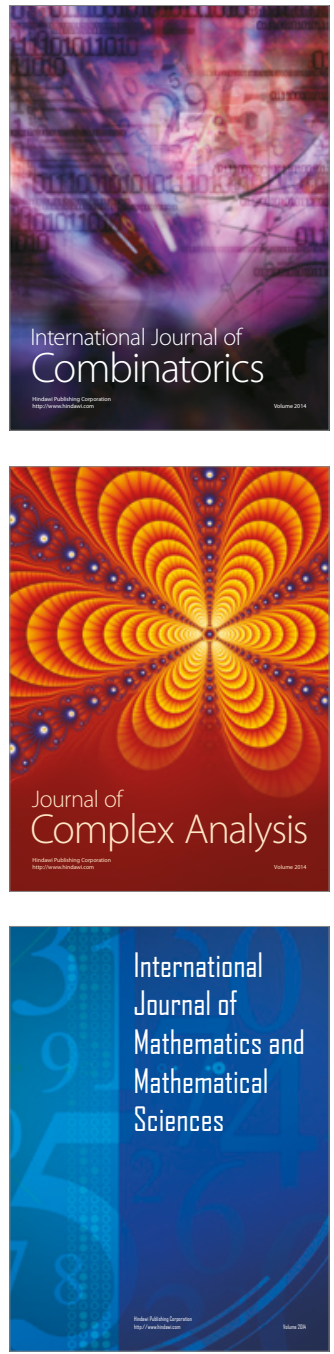


Algebra

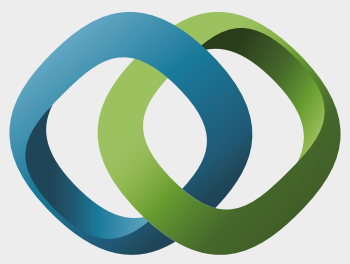

\section{Hindawi}

Submit your manuscripts at

https://www.hindawi.com
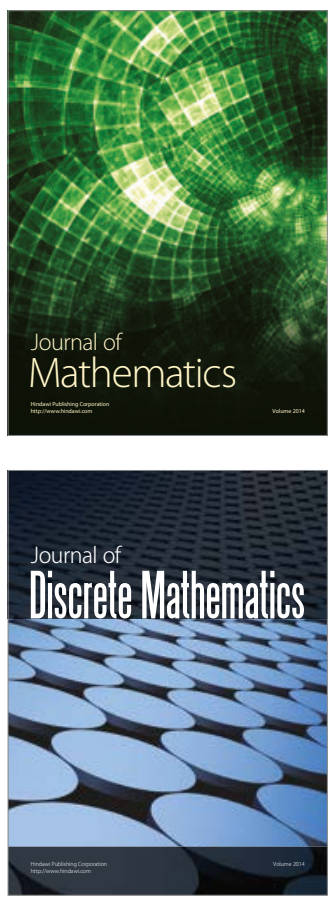

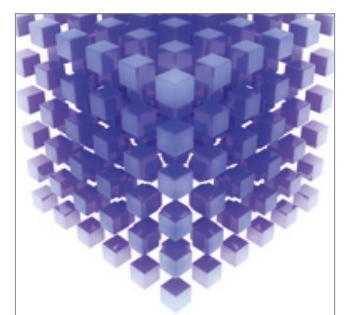

Mathematical Problems in Engineering
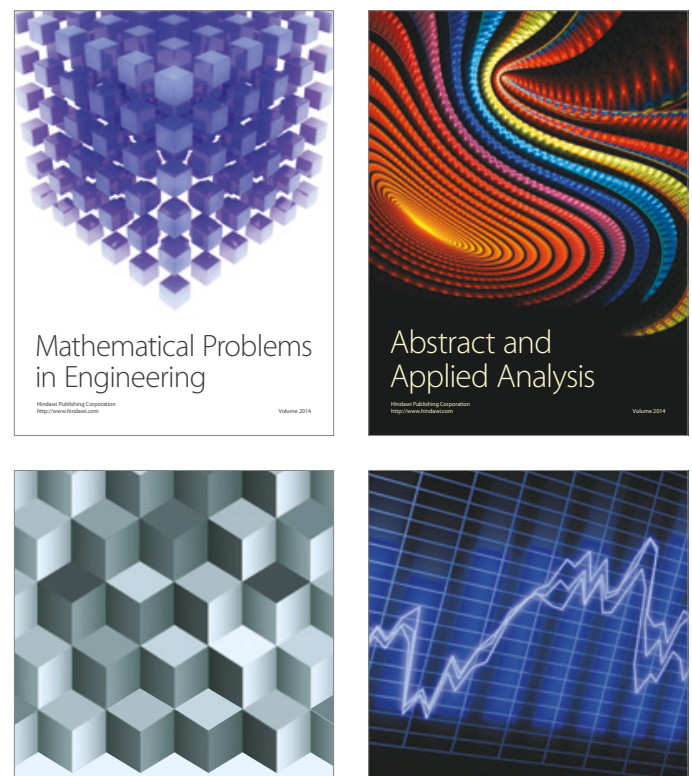

Journal of

Function Spaces

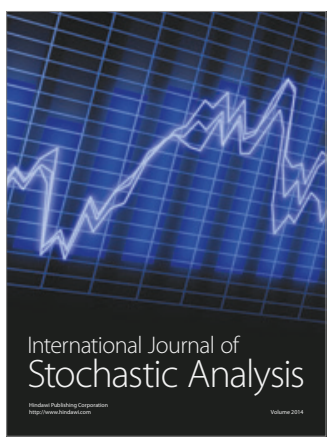

Probability and Statistics
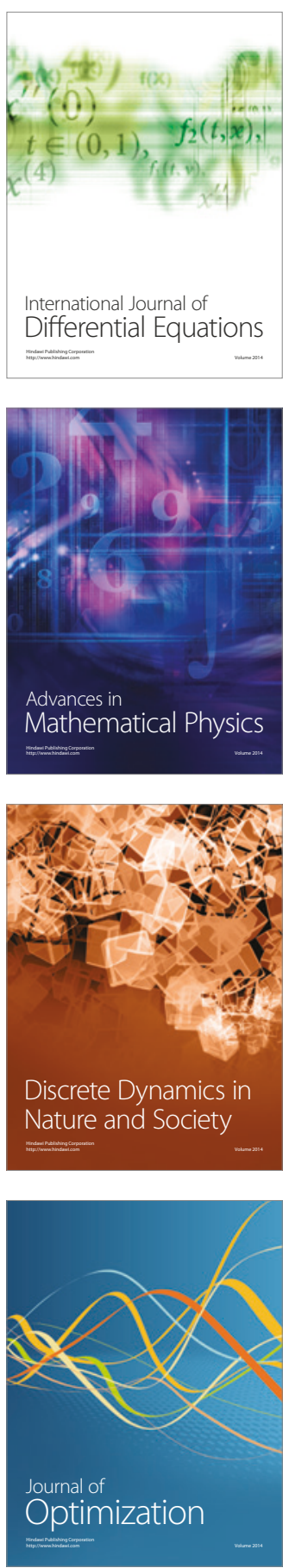